\title{
Remarks on the Generalized Chaplygin Gas
}

\author{
Rabin Banerjee ${ }^{1 *}$ Subir Ghosh ${ }^{2 \dagger}$ and Shailesh Kulkarni ${ }^{1 \dagger}$ \\ ${ }^{1}$ S. N. Bose National Centre for Basic Sciences, \\ JD Block, Sector III, Salt Lake, Kolkata-700098, India \\ ${ }^{2}$ Physics and Applied Mathematics Unit, Indian Statistical Institute, \\ 203 B.T Road, Kolkata 700108, India
}

\begin{abstract}
We have developed an action formulation for the Generalized Chaplygin Gas (GCG). The most general form for the nonrelativistic GCG action is derived consistent with the equation of state. We have also discussed a relativistic formulation for GCG by providing a detailed analysis of the Poincare algebra.
\end{abstract}

\section{Introduction}

The recent observation of accelerated expansion of the universe, concluded [1] from the study of luminosity of type Ia distant supernova, has put Cosmology in the center stage. Our inability to explain the origin of this expansion has led to the naming of this phenomenon as "Dark Energy" effect. The coinage obviously matches the other fuzzy area in Cosmology, i.e. the existence of "Dark Matter". There exist several plausible models at hand that can explain the astronomical data [1. The traditional one - vacuum energy or non-zero cosmological constant - fits well with the observational data. Unfortunately it is plagued with serious conceptual difficulties: smallness of the value of the cosmological constant in comparison with Planck mass scale and the coincidence problem, (that questions the reason for the near equality between energy densities of Dark Energy and dust-like matter in the present epoch), to name a few. The latter is circumvented by introducing scalar field (or Quintessence) models [2] inducing a dynamical vacuum energy, but only at the expense of fine tuning the scalar potential parameters. An alternative dynamical model [3] for Dark Energy, featuring Chaplygin Gas [4] or its generalization [5] - the Generalized Chaplygin Gas (GCG) - has created a lot of interest in recent times. Conventional analysis of the model [6] has attributed its matter density profile the flexibility to smoothly interpolate between a dust dominated era (at early times) to the Cosmological constant dominated era (at present times). A further generalization [7] to inhomogeneous GCG allows one to address the issue of Dark Matter as well. The GCG model has passed several experimental tests of various nature, such as high precision Cosmic Microwave Background Radiation data [8], supernova data 9] and gravitational lensing [10]. Naive analysis [11 seemed to suggest a disturbing phenomenon in the GCG model: possible existence of unphysical oscillations or even an exponential blow-up in the matter power spectrum at present. However, this problem has been solved in [12] by taking in to account the interaction between Dark Matter, Dark Energy and phantom-type Dark Energy [13]. (For a detailed exposition of these issues, see [14.)

The above mentioned ideal fluid system was introduced long ago by Chaplygin [4] as an effective model in computing the lifting force on a wing of an airplane. It obeys an exotic

\footnotetext{
*E-mail: rabin@bose.res.in

$\dagger$ E-mail: sghosh@isical.ac.in

‡E-mail: shailesh@bose.res.in
} 
equation of state,

$$
P=-\frac{B}{\rho}
$$

where $P(x)$ and $\rho(x)$ denote pressure and density respectively and $B$ a constant parameter. However, the interest in Chaplygin gas model actually goes beyond Cosmology (see [15] for a review, oriented towards the High Energy Physics community). It has a deep connection with the $D$-branes in a higher dimensional Nambu-Goto Formulation in light-cone parameterization [16]. It is also unique in admitting a supersymmetric generalization [17] for a fluid. The dynamical role of Chaplygin gas in cosmology has been shown in [18]. The above discussion clearly underlines the relevance and topicality of GCG models in Cosmology and High Energy Physics.

In the present work, we follow the same theme and focus our attention on the GCG models, where the generalization amounts to postulating the Chaplygin equation of state as,

$$
P=-\frac{B}{\rho^{\alpha}} ; \quad B>0 ; \quad 0<\alpha<1
$$

where the standard Chaplygin pressure equation (11) is recovered for $\alpha=1$. In the nonrelativistic regime, we have constructed the most general action for GCG consistent with (2). This construction has been done in two versions. In one case, the action involves the density $\rho$ and the velocity potential $\theta$. Elimination of $\rho$ is possible leading to the second version involving only $\theta$. This may be interpreted as a Born-Infeld type action in the nonrelativistic limit

Next, the construction of relativistic action for GCG is discussed. Here a Born-Infeld action involving only $\theta$ is proposed which has the correct nonrelativistic limit. Also for $\alpha=1$, it reproduces the standard Born-Infeld action for the Chaplygin gas. We may mention that our action is different from the one given in the literature [5]. Since the density plays an important role it becomes worthwhile to write the relativistic action for GCG involving both $\rho$ and $\theta$ analogous to the usual $\alpha=1$ case [15]. However here we are faced with certain problems. Our suggested form for GCG action has the correct nonrelativistic and $\alpha=1$ limits. However for $\alpha \neq 1$ it is relativistic only for large $\rho$. This is found by an explicit check of the Poincare algebra.

The paper is organized as follows: In Section 2 we provide a brief review of the non-relativistic and relativistic action formulations of the normal Chaplygin gas $(\alpha=1)$. This helps us to fix the notation and charts the course of our subsequent analysis in the paper. Section 3 comprises an analogous study for the GCG $(\alpha \neq 1)$ and introduces new expressions for the nonrelativistic GCG action. Section 4 is devoted to the construction and subsequent analysis of the relativistic GCG. In Section 5 we provide our conclusion and propose avenues for future study.

\section{Normal $(\alpha=1)$ Chaplygin gas : a brief review}

Before concentrating on the Chaplygin gas, let us discuss some basic notions of fluid dynamics, in the Lagrangian (action) formulation [15].

We start with the non-relativistic scenario. The equations of motion, governing an ideal fluid in arbitrary space dimensions, are given by

$$
\begin{aligned}
\partial_{t} \rho(t, \mathbf{x})+\nabla \cdot(\rho(t, \mathbf{x}) \mathbf{v}(t, \mathbf{x})) & =0 \\
\partial_{t} \mathbf{v}(t, \mathbf{x})+\mathbf{v}(t, \mathbf{x}) \cdot \nabla \mathbf{v}(t, \mathbf{x}) & =\mathbf{f}(t, \mathbf{x}),
\end{aligned}
$$

where $\rho(t, \mathbf{x})$ and $\mathbf{v}(t, \mathbf{x})$ are the matter density and velocity fields respectively. The first identity reflects the matter conservation and the second is the Euler equation of motion. We consider the motion of fluid to be isentropic. Hence the force $f$ is derived from a $\rho$-dependent potential $V(\rho)$,

$$
\mathbf{f}=-\nabla V^{\prime}(\rho) \text {. }
$$


Here prime denotes derivative with respect to $\rho$. Note that $V^{\prime}(\rho)$ is the enthalpy. The all important relation connecting $V$ with the pressure $P$ is given by,

$$
P(\rho)=\rho V^{\prime}(\rho)-V(\rho) .
$$

In the case of irrotational fluid we can further restrict the velocity to be a gradient of some scalar field $\theta(\mathbf{x})$,

$$
\mathbf{v}=\nabla \theta
$$

The (non-relativistic) Hamiltonian for irrotational motion is just the sum of kinetic and potential energy,

$$
H=\int d r\left(\frac{1}{2} \rho\left(\partial_{i} \theta\right)^{2}+V(\rho)\right)
$$

Now, the first order form of the Lagrangian $L$, corresponding to (요 is given by,

$$
L=\int d r\left(\theta \dot{\rho}-\frac{1}{2} \rho\left(\partial_{i} \theta\right)^{2}-V(\rho)\right) .
$$

From the symplectic structure it is clear that $\rho$ and $\theta$ are conjugate variables, satisfying the the canonical Poisson bracket,

$$
\{\theta(\mathbf{x}), \rho(\mathbf{y})\}=\delta(\mathbf{x}-\mathbf{y})
$$

The nature of the potential function $V(\rho)$ will specify the particular fluid model under study. For Chaplygin gas the potential profile is given by,

$$
V(\rho)=\frac{\lambda}{\rho}
$$

where $\lambda$ is the interaction strength. Using (7) the Lagrangian for the Chaplygin gas model is given by

$$
L=\int d r\left(\theta \dot{\rho}-\frac{1}{2} \rho\left(\partial_{i} \theta\right)^{2}-\frac{\lambda}{\rho}\right),
$$

and the Bernoulli equation follows:

$$
\dot{\theta}+\frac{1}{2}\left(\partial_{i} \theta\right)^{2}=\frac{\lambda}{\rho^{2}}
$$

It is possible to eliminate $\rho$ from the Lagrangian to obtain a non-relativistic Born-Infeld like structure in $\theta$,

$$
L(\theta)=-2 \sqrt{\lambda} \int d r \sqrt{\left(\dot{\theta}+\frac{1}{2}\left(\partial_{i} \theta\right)^{2}\right)}
$$

with the equation of motion,

$$
\partial_{t}\left(\dot{\theta}+\frac{1}{2}\left(\partial_{i} \theta\right)^{2}\right)^{-\frac{1}{2}}+\partial_{i}\left[\partial_{i} \theta\left(\dot{\theta}+\frac{1}{2}\left(\partial_{i} \theta\right)^{2}\right)^{-\frac{1}{2}}\right]=0 .
$$

Now we come to the relativistic generalization of Chaplygin gas [15]. A Lagrangian has been suggested for the normal $(\alpha=1)$ Chaplygin gas in [15],

$$
L=\int d r\left(\theta \dot{\rho}-\sqrt{\left(\rho^{2} c^{2}+a^{2}\right)} \sqrt{c^{2}+\left(\partial_{i} \theta\right)^{2}}\right)
$$

where $a$ is a parameter in the theory. Although (16) does not have a manifestly relativistic form, its Poincare invariance has been demonstrated explicitly in [18] in a Hamiltonian framework 
(see Section 4 of the present paper as well). On the other hand, one can eliminate $\rho$ once again from (16) to obtain the Born-Infeld form,

$$
L=-a \int d r \sqrt{c^{2}-\partial_{\mu} \theta \partial^{\mu} \theta}
$$

which is manifestly relativistic.

To get the correct non-relativistic limit, one has to consider the map [15],

$$
\theta \rightarrow \theta-t c^{2}
$$

Under this transformation, the relativistic model (16) will reduce to the non-relativistic one in

(12) with the identification $\lambda \equiv \frac{a^{2}}{2}$. This concludes our review [15] of action formulation of Chaplygin gas.

\section{$3 \quad$ Nonrelativistic generalized $(\alpha \neq 1)$ Chaplygin gas}

In GCG the equation of state (1) is replaced by a more flexible one, given in (2). In order to incorporate this generalization in the action formulation, our starting point is to find a suitable potential $V(\rho)$ compatible with (2). To find the general solution for $V(\rho)$ we start from an ansatz,

$$
V(\rho)=\left(\frac{B}{\alpha+1}\right) \frac{1}{\rho^{\alpha}}+u(\rho)
$$

where $u(\rho)$ is such that $V(\rho)$ satisfies

$$
-\frac{B}{\rho^{\alpha}}=\rho \frac{d V(\rho)}{d \rho}-V(\rho) .
$$

This follows from (2) and the enthalpy relation (6). This implies $u(\rho)$ must satisfy

$$
\rho \frac{d u}{d \rho}-u=0
$$

The solution for above equation is

$$
u(\rho)=I \rho .
$$

where $I$ is an integration constant.

Hence the most general form of the potential $V(\rho)$ is

$$
V(\rho)=\left(\frac{B}{\alpha+1}\right) \frac{1}{\rho^{\alpha}}+I \rho .
$$

For the irrotational fluid we can write the Hamiltonian (8), with $V(\rho)$ as given in (23)

$$
H=\int d r\left(\frac{1}{2} \rho\left(\partial_{i} \theta\right)^{2}+\frac{B}{(\alpha+1) \rho^{\alpha}}+I \rho\right),
$$

Now the first order form of the Lagrangian follows from (24),

$$
L^{\alpha}=\int d r\left(\theta \dot{\rho}-\frac{1}{2} \rho\left(\partial_{i} \theta\right)^{2}-\frac{B}{(\alpha+1) \rho^{\alpha}}-I \rho\right)
$$

where the superscript $\alpha$ on $L$ reveals the fact that we are dealing with GCG. 
Variation of $\rho$ yields the Bernoulli equation,

$$
\dot{\theta}+\frac{1}{2}\left(\partial_{i} \theta\right)^{2}=\frac{B \alpha}{(\alpha+1) \rho^{\alpha+1}}-I .
$$

To obtain the $\rho$ independent Lagrangian for GCG, one can use the Bernoulli equation to reexpress $\rho$ in terms of $\theta$,

$$
\rho=\left(\frac{\alpha B}{\alpha+1}\left(\dot{\theta}+\frac{1}{2}\left(\partial_{i} \theta\right)^{2}+I\right)^{-1}\right)^{\frac{1}{\alpha+1}} .
$$

It is very convenient to rewrite the Lagrangian given in (25) in the following form

$$
L^{\alpha}=-\int d r\left(\dot{\theta} \rho+\frac{\rho}{2}\left(\partial_{i} \theta\right)^{2}+\frac{B}{(\alpha+1) \rho^{\alpha}}+I \rho\right) .
$$

In the above equation we have omitted total derivative terms. Substituting $\rho$ from (27) in (28) we find,

$$
L^{\alpha}(\theta)=-\left(\frac{\alpha}{\alpha+1}\right)^{\frac{\alpha}{\alpha+1}} B^{\frac{1}{\alpha+1}} \int d r \sqrt{\left(\dot{\theta}+\frac{1}{2}\left(\partial_{i} \theta\right)^{2}+I\right)^{\frac{2 \alpha}{\alpha+1}}} .
$$

This is the most general form of GCG Lagrangian and is a central result of this paper. It is the Born-Infeld version of nonrelativistic GCG.

The equation of motion for $\theta$ turns out to be,

$$
\partial_{t}\left(\dot{\theta}+\frac{1}{2}\left(\partial_{i} \theta\right)^{2}\right)^{\frac{-1}{\alpha+1}}+\partial_{i}\left[\partial_{i} \theta\left(\dot{\theta}+\frac{1}{2}\left(\partial_{i} \theta\right)^{2}+I\right)^{\frac{-1}{\alpha+1}}\right]=0 .
$$

A definite simplification occurs by setting $I=0$. Then the Lagrangians (25) and (29) reduce to

$$
\begin{gathered}
L^{\alpha}=\int d r\left(\theta \dot{\rho}-\frac{1}{2} \rho\left(\partial_{i} \theta\right)^{2}-\frac{B}{(\alpha+1) \rho^{\alpha}}\right), \\
L^{\alpha}(\theta)=-\left(\frac{\alpha}{\alpha+1}\right)^{\frac{\alpha}{\alpha+1}} B^{\frac{1}{\alpha+1}} \int d r \sqrt{\left(\dot{\theta}+\frac{1}{2}\left(\partial_{i} \theta\right)^{2}\right)^{\frac{2 \alpha}{\alpha+1}}} .
\end{gathered}
$$

Putting $\alpha=1$ in (31) and (32) reproduces the expressions for the usual Chaplygin gas [15].

\section{Relativistic generalized Chaplygin gas}

Now we turn to the relativistic form of GCG. Any relativistic version of GCG must satisfy two conditions: it should have the correct nonrelativistic limit (25) or (29), secondly, for $\alpha=1$ it should reduce to (12) or (14).

To begin with we suggest a manifestly Poincare invariant model for GCG, given by

$$
L^{\alpha}=-\left(a^{\prime}\right)^{\frac{1}{\alpha+1}} \int d r \sqrt{\left(c^{2}-\partial_{\mu} \theta \partial^{\mu} \theta\right)^{\frac{2 \alpha}{\alpha+1}}}
$$

In the nonrelativistic limit it agrees with (32). To show this we exploit (18) and use the fact that $\partial_{\mu} \theta \partial^{\mu} \theta=\frac{\dot{\theta}^{2}}{c^{2}}-\partial_{i} \theta^{2}$, to simplify the above Lagrangian,

$$
L^{\alpha}=-\left(a^{\prime}\right)^{\frac{1}{\alpha+1}} \int d r\left[-\frac{\dot{\theta}}{c^{2}}+2 \dot{\theta}+\left(\partial_{i} \theta\right)^{2}\right]^{\frac{\alpha}{\alpha+1}} .
$$


Now taking the large $c$ limit we get

$$
\lim _{c \rightarrow \infty} L^{\alpha}=-(2)^{\frac{\alpha}{\alpha+1}}\left(a^{\prime}\right)^{\frac{1}{\alpha+1}} \int d r \sqrt{\left[\dot{\theta}+\left(\frac{\left.\partial_{i} \theta\right)^{2}}{2}\right]^{\frac{2 \alpha}{\alpha+1}}\right.} .
$$

By identifying $a^{\prime}=\left(\frac{\alpha}{2(\alpha+1)}\right)^{\alpha} B$ we see that (35) agrees with (32). Also, in the $\alpha=1$ limit our Lagrangian (33) reduces to that of usual relativistic Chaplygin gas (17). This shows that it is possible to interpret (33) as a viable form for the relativistic GCG Lagrangian.

At this point we should mention that there exists in the literature a Poincare invariant form for GCG [5]

$$
L_{b}=-A^{\frac{1}{1+\alpha}} \int d r\left[c^{2}-\left(\partial_{\mu} \theta \partial^{\mu} \theta\right)^{\frac{1+\alpha}{2 \alpha}}\right]^{\frac{\alpha}{\alpha+1}} .
$$

Note that, for $\alpha \neq 1$ the above Lagrangian is different from (33). However for $\alpha=1$ it agrees with normal Chaplygin gas Lagrangian [15]. GCG of similar nature [5] coupled to gravity has been considered in [19].

Now consider the nonrelativistic limit of (36) $)$. Following the same procedure as discussed above we get in this limit

$$
L_{b}=-A^{\frac{1}{1+\alpha}}(2 Y)^{\frac{\alpha}{\alpha+1}} \sqrt{\left[\frac{Z}{2 Y}+\left(\dot{\theta}+\frac{1}{2} \nabla \theta^{2}\right)\right]^{\frac{2 \alpha}{1+\alpha}}} .
$$

where

$$
\begin{aligned}
& Y=\frac{1+\alpha}{2 \alpha} c^{\frac{1+\alpha}{\alpha}} \\
& Z=c^{2}-c^{\frac{1+\alpha}{\alpha}} .
\end{aligned}
$$

This Lagrangian is same as that of (29) provided we identify $I$ with $\frac{Z}{2 Y}$. Thus both (33) and (36) are valid forms for the relativistic GCG whose nonrelativistic limits correspond to different parametrizations of the general form for nonrelativistic GCG given in (29).

Let us next attempt to construct the relativistic GCG model by including the density field $\rho$. Also, since the density field plays an important role in the observational analysis of GCG it is worthwhile to have a relativistic version for GCG involving $\rho$ and the velocity potential $\theta$.

To this end, we consider the following Lagrangian for relativistic GCG:

$$
L^{\alpha}=\int d r\left(\theta \dot{\rho}-\sqrt{\left(\rho^{2} c^{2}+\frac{a^{2}}{\rho^{\alpha-1}}\right)} \sqrt{c^{2}+\left(\partial_{i} \theta\right)^{2}}\right),
$$

where $a$ is a constant parameter. To ensure the correct nonrelativistic limit we use the same map as (18), and explicitly check that in the $c \rightarrow \infty$ limit, the nonrelativistic GCG model (31) is reproduced, provided we identify,

$$
a=\sqrt{\frac{2 B}{\alpha+1}} .
$$

We put $c=1$ and obtain the equations of motion,

$$
\begin{gathered}
\dot{\rho}+\partial_{i}\left(\frac{\sqrt{\left(\rho^{2}+\frac{a^{2}}{\rho^{\alpha-1}}\right)}}{\sqrt{1+\left(\partial_{i} \theta\right)^{2}}} \partial_{i} \theta\right)=0, \\
\dot{\theta}=-\frac{\sqrt{1+\left(\partial_{i} \theta\right)^{2}}}{\sqrt{\left(\rho^{2}+\frac{a^{2}}{\rho^{\alpha-1}}\right)}}\left[\rho c^{2}-\left(\frac{\alpha-1}{2}\right) \frac{a^{2}}{\rho^{\alpha}}\right] .
\end{gathered}
$$


They also have the correct $\alpha=1$ limit [15].

As we have pointed out before, the Lagrangian (38) has been posited by us in analogy with the relativistic Lagrangian given in (16) [15] and we know that (38) has the correct $\alpha=1$ limit. Since the model (38) is not manifestly Lorentz invariant, it becomes imperative to check the Poincare algebra. To this end, we follow our previous work [18] and compute the canonical energy-momentum tensor $T_{\mu \nu}$ (in the Noether prescription),

$$
T_{\mu \nu}=\frac{\partial \mathcal{L}}{\partial\left(\partial^{\mu} \psi_{i}\right)} \partial_{\nu} \psi^{i}-g_{\mu \nu} \mathcal{L}
$$

Using the above definition, the explicit form of the components of $T_{\mu \nu}$ are given by,

$$
\begin{aligned}
& T_{00}=\sqrt{\left(\rho^{2}+\frac{a^{2}}{\rho^{\alpha-1}}\right)} \sqrt{1+\left(\partial_{i} \theta\right)^{2}} \\
& T_{0 i}=\theta \partial_{i} \rho \\
& T_{i 0}=-\frac{\sqrt{\rho^{2}+\frac{a^{2}}{\rho^{\alpha-1}}}}{\sqrt{1+\left(\partial_{k} \theta\right)^{2}}}\left(\partial_{i} \theta\right) \dot{\theta}=\left(\rho(\mathbf{x})+\left(\frac{1-\alpha}{2}\right) \frac{a^{2}}{\rho^{\alpha}(\mathbf{x})}\right) \partial_{i} \theta, \\
& T_{i j}=-\frac{\sqrt{\rho^{2}+\frac{a^{2}}{\rho^{\alpha-1}}}}{\sqrt{1+\left(\partial_{k} \theta\right)^{2}}}\left(\partial_{i} \theta\right)\left(\partial_{j} \theta\right)-g_{i j} \mathcal{L}^{\alpha} .
\end{aligned}
$$

Notice that $T_{0 i} \neq T_{i 0}$. Using the equations of motion (40), (41) one can explicitly verify the conservation law,

$$
\partial^{\mu} T_{\mu \nu}=0
$$

Hence $T_{\mu \nu}$ is a conserved but non-symmetric energy-momentum tensor.

Once we have the forms of $T_{00}$ and $T_{0 i}$ we can easily obtain the expression for the momenta $P_{\mu}$ and the angular momenta $M_{\mu \nu}$. They are related to the components of the energy-momentum tensor as

$$
\begin{aligned}
P_{\mu} & =\int d^{3} x T_{0 \mu}, \\
M_{\mu \nu} & =\int d^{3} x\left(T_{0 \mu} x_{\nu}-T_{0 \nu} x_{\mu}\right) .
\end{aligned}
$$

By using (43) and (44) we get,

$$
\begin{aligned}
P_{0} & =\int d^{3} x \sqrt{\left(\rho^{2}+\frac{a^{2}}{\rho^{\alpha-1}}\right)} \sqrt{1+\left(\partial_{i} \theta\right)^{2}} \\
P_{i} & =\int d^{3} x \theta \partial_{i} \rho \\
M_{0 i} & =\int d^{3} x \sqrt{\left(\rho^{2}+\frac{a^{2}}{\rho^{\alpha-1}}\right)} \sqrt{1+\left(\partial_{i} \theta\right)^{2}} x_{i}-\theta \partial_{i} \rho x_{0}, \\
M_{i j} & =\int d^{3} x\left(\theta \partial_{i} \rho x_{j}-\theta \partial_{j} \rho x_{i}\right) .
\end{aligned}
$$

Using the Poisson bracket (10) we are able to compute the following algebra,

$$
\begin{aligned}
\left\{M_{i j}, M_{k l}\right\} & =\left(g_{j k} M_{i l}-g_{i k} M_{j l}-g_{i l} M_{k j}+g_{j l} M_{k i}\right) \\
\left\{M_{o i}, M_{k l}\right\} & =\left(g_{i k} M_{0 l}-g_{i l} M_{0 k}\right) \\
\left\{M_{0 i}, M_{o j}\right\} & =-g_{00} \int d^{3} x\left(\theta \partial_{i} \rho x_{j}-\theta \partial_{j} \rho x_{i}\right)\left(1-\alpha\left(\frac{1-\alpha}{2}\right) \frac{a^{2}}{\rho^{\alpha+1}}\right) .
\end{aligned}
$$


Similarlly the algebra between $P_{\mu}-M_{\mu \nu}$ is given by

$$
\begin{aligned}
& \left\{M_{0 i}, P_{j}\right\}=P_{0} g_{i j}, \\
& \left\{M_{i j}, P_{k}\right\}=g_{j k} P_{i}-g_{i k} P_{j}, \\
& \left\{M_{0 i}, P_{0}\right\}=-g_{00} \int d^{3} x \theta \partial_{i} \rho\left(1-\alpha\left(\frac{1-\alpha}{2}\right) \frac{a^{2}}{\rho^{\alpha+1}}\right) .
\end{aligned}
$$

Finally, the algebra between $P_{\mu}-P_{\nu}$ is found out to be,

$$
\left\{P_{\mu}, P_{\nu}\right\}=0
$$

Concentrate on the two Poisson brackets (56) and (59). We find that for $\alpha=1$ the complete Poincare algebra is satisfied. This corresponds to the usual Chaplygin Poincare algebra [18]. However for $\alpha \neq 1$ (which corresponds to the GCG model) the Poincare algebra closes only in the large density limit $(\rho>>1)$. It is, however, reassuring to note that the Schwinger condition,

$$
\left\{T_{00}(\mathbf{x}), T_{00}(\mathbf{y})\right\}=\left(T_{i 0}(\mathbf{x})+T_{i 0}(\mathbf{y})\right) \partial_{i}^{x} \delta(\mathbf{x}-\mathbf{y}),
$$

is satisfied for any $\alpha$ and $\rho$.

\section{Conclusions:}

To conclude, we have studied various aspects of the Generalized Chaplygin Gas (GCG) models. In the nonrelativistic regime, we have constructed a general form of the Lagrangian for GCG, that obeys the generalized equation of state. Different parameterizations of this master Lagrangian yield different inequivalent models for GCG, such as the one studied here and the one in [5]. In this sense the construction of nonrelativistic GCG is not unique. Naturally, the same conclusion extends for a relativistic formulation of GCG.

For the relativistic scenario, we have proposed a Born-Infeld like model for GCG, which in the nonrelativistic limit, reduces to the conventional GCG. However, unlike the usual $\alpha=1$ Chaplygin gas case, the construction of a relativistic GCG model, including both density field and velocity potential is nontrivial. In this context, our model reduces to the usual one, quoted in literature [15] for $\alpha=1$ and also has the correct nonrelativistic limit. However the Poincare algebra closes only in the limit of large density.

The relativistic Lagrangian formulation for GCG, initiated here, opens up a host of avenues for future study. First, we wish to extend our previous study 18 of the Chaplygin matter in FRW spacetime, to the case of a GCG, to observe its cosmological implications. Next, our aim is to analyse symmetry properties of GCG (first elucidated in [15] for usual Chaplygin gas), as well as its connection to D-branes.

\section{References}

[1] A.Reiss et.al., Astron.J., 116 (1998)1009; S.J.Perlmutter et.al., Astroph.J., 517 (1999)565; N.A.Bahcall et.al., Science, 284 (1999)1481.

[2] B.Ratra and P.J.E.Peebles, Phys.Rev. D37 (1988)3406; R.R.Caldwell, R.Dave and P.J.Steinhardt, Phys.Rev.Lett. 80 (1998)1582 (astro-ph/9708069).

[3] A.Kamenshchik, U.Moschella and V.Pasquier, Phys.Lett. B511 (2001)265 (gr-qc/0103004).

[4] S.Chaplygin, Sci. Mem. Moscow Univ. Math. Phys. 21 (1904)1.

[5] M.C.Bento, O.Bertolami and A.A.Sen, Phys.Rev. D66 (2002)043507 (gr-qc/0202064). 
[6] A.Frolov, L.Kofman and A.Starobinski, Phys.Lett. B545 (2002)8 (hep-th/0204187); U.Debnath, A.Banerjee, S.Chakraborty, Class.Quant.Grav. $21 \quad(2004) \quad 5609$ (gr-qc/0411015); Zong-Hong Zhu Astron.Astrophys. 423 (2004) 421 (astro-ph/0411039); A.A.Sen and R.J.Scherrer, astro-ph/0507717; M. K. Mak, T. Harko, Phys.Rev. D71 (2005) 104022 (gr-qc/0505034); V.Gorini, A.Kamenshchik, U.Moschella, V.Pasquier, A.Starobinsky, astro-ph/0504576; S. Capozziello, V.F. Cardone, A. Troisi, Phys.Rev. D71 (2005) 043503; L.P.Chimento, R.Lazkoz, astro-ph/0505254; Varun Sahni, astro-ph/0403324; O.Bertolami, P.T.Silva, astro-ph/0507192; B. Mota, M. Makler, M.J. Reboucas, astro-ph/0506499; Dao-jun Liu, Xin-zhou Li, Chin.Phys.Lett. 22 (2005) 1600; Yungui Gong, JCAP 0503 (2005) 007.

[7] N.Bilic, G.B.Tupper and R.Viollier, Phys.Lett. B535 (2002) 17 (astro-ph/0111325).

[8] M.C.Bento, O.Bertolami and A.A.Sen, Phys.Lett. B575 (2003)172 (astro-ph/0303538); Phys.Rev. D67 (2003)063003 (astro-ph/0210468); L.Amendola et.al., JCAP 0307 (2003)005.

[9] J.C.Fabris, S.B.V Goncalves and P.E.de Souza, astro-ph/0207430; A.Dev, J.S.Alcaniz and D.Jain, Phys.Rev. D67 (2003)023515 (astro-ph/0209379); V.Gorini, A.Kamenshchik and U.Moschella, Phys.Rev. D67 (2003)063509 (astro-ph/0209395).

[10] P.T.Silva and O.Bertolami, Ap.J. 500 (2003)829; A.Dev, D.Jain and J.S.Alcaniz, Astron.Astrophys. 417 (2004)847 (astro-ph/0311056).

[11] H.Sandvik, M.Tegmark, M.Zaldarriga and I.Waga, Phys.Rev. D69 (2004)123524 (astro-ph/0212114).

[12] M.C.Bento, O.Bertolami and A.A.Sen, Phys.Rev. D70 (2004)083519 (astro-ph/0407239).

[13] Xin Zhang, Feng-Quan Wu and Jingfei Zhang, JCAP 0601 (2006) 003 (astro-ph/0411221).

[14] O. Bertolami, (astro-ph/0504275).

[15] D.Bazeia and R. Jackiw, Ann.Phys. 270 (1998)246 (hep-th/9803165); D.Bazeia, Phys.Rev. D59 (1999)085007; also see the review by R. Jackiw, V.P.Nair, S.-Y.Pi and A.P.Polychronakos, J.Phys. A37 (2004) R327 (hep-th/0407101).

[16] M.Bordemann and J.Hopp, Phys.Lett. B317 (1993)315 (hep-th/9307036).

[17] R.Jackiw and A.P.Polychronakos, Phys.Rev. D62 (2000)085019 (hep-th/0004083).

[18] R.Banerjee and S.Ghosh, Mod.Phys.Lett. A21 (2006) 1511 (gr-qc/0508021).

[19] M. Hassaine, J. Math. Phys. 47 (2006)033101 (hep-th/0511243). 